\title{
COEFFICIENTS ASSOCIATED WITH THE EXPANSION OF CERTAIN PRODUCTS
}

\author{
D. P. ROSELLE ${ }^{1}$
}

ABSTRACT. Simple combinatorial interpretations are given for the coefficients of the polynomials $H_{n}(x, y)$ and $G_{n}(x, y)$ defined by $\Pi\left(1+x^{n} y^{m} t\right)$ $=\sum G_{n}(x, y) t^{n} /(x){ }_{n}(y){ }_{n}$ and $\Pi\left(1-x^{n} y^{m} t\right)^{-1}=\sum H_{n}(x, y) t^{n} /(x){ }_{n}(y){ }_{n}$.

1. Introduction. Consider the products

and

$$
\prod_{m, n=0}^{\infty}\left(1+x^{n} y^{m} t\right)=\sum_{n=0}^{\infty} G_{n}(x, y) \frac{t^{n}}{(x)_{n}(y)_{n}}
$$

where

$$
\prod_{m, n=0}^{\infty}\left(1-x^{n} y^{m} t\right)^{-1}=\sum_{n=0}^{\infty} H_{n}(x, y) \frac{t^{n}}{(x)_{n}(y)_{n}}
$$

$$
(x)_{n}=(1-x)\left(1-x^{2}\right) \ldots\left(1-x^{n}\right), \quad(x)_{0}=1 .
$$

The products (1.1) and (1.2) were first considered by Carlitz [2] and Wright [7] in response to a research problem posed by Bellman [1]. They show that $G_{n}(x, y)$ and $H_{n}(x, y)$ are polynomials in $x$ and $y$ with integral coefficients of degree $1 / 2 n(n-1)$ in each variable and that

$$
H_{n}(x, y)=y^{n(n-1) / 2} G_{n}(x, 1 / y) \text {. }
$$

Wright conjectured [7], [8] that the coefficients of these polynomials are nonnegative integers. This conjecture was settled by Gordon [4] who, using a combinatorial argument, proved this conjecture and the analogous result for the more general product

$$
\prod_{k_{j}=0}\left(1-x_{1}^{k} \ldots x_{s}^{k} s^{-1}\right. \text {. }
$$

Here we supply a simple combinatorial meaning for the coefficients of

Received by the editors February 13, 1973 and, in revised form, August 17, 1973.

AMS (MOS) subject classifications (1970). Primary 05A15, 05 A17.

1 Supported in part by National Science Foundation Grant GP-19207. 
the polynomials $H_{n}(x, y)$ and $G_{n}(x, y)$. Let $\pi=\left(i_{1}, i_{2}, \cdots, i_{n}\right)$ be a permutation of $Z_{n}=\{1,2, \cdots, n\}$. We say that $\pi$ has $r$ rises and $s$ readings if there are $r-1$ subscripts $j$ such that $i_{j}<i_{j+1}$ and if it is necessary to make $s$ left to right scans of $\pi$ in order that the elements are arranged in the order $1,2, \cdots, n$. Thus, for example, the permutation $\pi=(216354)$ has 3 rises and 4 readings. The number, $R_{n}(r, s)$, of permutations of $Z_{n}$ with $r$ rises and $s$ readings has been studied [3] and suggests the concepts of weighted rises and weighted readings which are of interest here.

We say that the permutation $\pi=\left(i_{1}, i_{2}, \ldots, i_{n}\right)$ has $u$ weighted rises and $v$ weighted readings if each rise position $i_{j}<i_{j+1}$ is assigned the weight $n-j$ and, if the element $i$ is read on the $j$ th scan, then it is assigned the weight $s_{i}=j-1$ and

$$
\sum_{i_{j}<i}(n-j)=u, \quad \sum_{i=1}^{n} s_{i}=v .
$$

Thus, continuing the earlier example, the permutation $\pi=(216354)$ has $u=$ $4+2=6$ weighted rises and $v=0 \cdot 1+1 \cdot 3+2 \cdot 1+3 \cdot 1=8$ weighted readings. We remark that a permutation $\pi$ has $v$ weighted readings implies that $\pi^{-1}$ has $\left(\begin{array}{l}n \\ 2\end{array}\right)-v$ weighted rises.

Let $c_{n}(u, v)$ denote the number of permutations of $Z_{n}$ with $u$ weighted rises and $v$ weighted readings. The main result of this paper is to show that

$$
G_{n}(x, y)=\sum_{u, v=0}^{\left(\begin{array}{c}
n \\
2
\end{array}\right)} c_{n}(u, v) x^{u} y^{u} \text {. }
$$

It follows at once from (1.3), (1.5) and our earlier remark that

$$
H_{n}(x, y)=\sum_{u, v=0}^{\left(\begin{array}{c}
n \\
2
\end{array}\right)} d_{n}(u, v) x^{u} y^{v},
$$

where $d_{n}(u, v)$ denotes the number of permutations of $Z_{n}$ with $u$ weighted rises whose inverses have $v$ weighted rises.

It is possible to give an alternative combinatorial description of these coefficients. Namely, $d_{n}(u, v)$ is equal to the number of pairs $\left\{\left(a_{1}, a_{2}, \ldots, a_{n}\right)\right.$, $\left.\left(b_{1}, \ldots, b_{n}\right)\right\}$ of $n$-tuples of positive integers satisfying

(i) for each $i \geqq 1$ and $i \in\{1, \ldots, n\}$, the number of occurrences of $i$ among $a_{1}, a_{2}, \cdots, a_{j}$ (respectively, $b_{1}, b_{2}, \ldots, b_{j}$ ) is at least as large as the number of occurrences of $i+1$ among $a_{1}, a_{2}, \ldots, a_{j}$ (respectively, $b_{1}$, $b_{2}, \ldots, b_{j}$ );

(ii) for each $i$, the number of $a_{j}$ 's which are equal to $i$ is the same as the number of $b_{k}$ 's which are equal to $i$; 
(iii) $\Sigma\left\{i \mid a_{i}<a_{i+1}\right\}=u, \Sigma\left\{j \mid b_{j}<b_{j+1}\right\}=v$.

For example, $d_{5}(4,5)=4$ corresponds to the four pairs

$$
\text { (12121), (12112); (12131), (12113); (12131), (11231); (12132), (11232). }
$$

The equivalence of these two interpretations can be established direct$\mathrm{ly}$, but it is of interest to note that the second follows from some recent work of Stanley in the theory of plane partitions. A sketch of the details involved begins by noting that if $g_{t}(x)$ is the polynomial defined by (7) [6], then $H_{n}(x, x)=g_{n}(x)$ : Next, a generalization of Theorem 5.1 of [6] shows that

$$
x^{n} y^{n} H_{n}(x, y)=(x)_{n}(y){ }_{n} \sum_{\lambda} F(\lambda, x) F(\lambda, y) .
$$

Applying the result of Corollary 7.2 of [5], we are able to deduce the second interpretation for the coefficients $d_{n}(u, v)$.

The author is indebted to the referee for pointing out the second combinatorial interpretation.

2. The main result. It follows from (1.1) that

$$
\sum_{n=0}^{\infty} G_{n}(x, y) \frac{t^{n}}{(x)_{n}(y)_{n}}=\sum_{n, k, m=0}^{\infty} t^{n} x^{k} y^{m} N_{n}(k, m),
$$

where $N_{n}(k, m)$ denotes the number of partitions of the bipartite $(k, m)$ of the form

$$
k=a_{1}+\cdots+a_{n}, \quad m=b_{1}+\cdots+b_{n},
$$

where the $a_{i}$ and $b_{i}$ are nonnegative integers subject to the conditions $a_{1} \leqq$ $a_{2} \leqq \cdots \leqq a_{n}$ and if $a_{i}=a_{i+1}$ then $b_{i} \neq b_{i+1}$. We can then arrange the parts of the partition so that if $a_{i}=a_{i+1}$ then $b_{i}>b_{i+1}$.

In order to prove (1.5), we first note that, using (2.1), we have the equivalent formulation

$$
N_{n}(k, m)=\sum_{u, v=0}^{\left(\begin{array}{c}
n \\
2
\end{array}\right)} c_{n}(u, v) p_{n}(k-u) p_{n}(m-v),
$$

where $p_{n}(j)$ is the number of partitions of the integer $j$ in $n$ nonnegative integers. We now give a constructive proof that $(2.2)$ holds. Note that we assume that $p_{n}(j)=0$ if $j<0$.

First suppose that $\pi=\left(i_{1}, i_{2}, \ldots, i_{n}\right)$ has rise positions $j_{1}, j_{2}, \ldots, j_{r}$; that is, $i_{j_{k}}<i_{j_{k}+1}$. Associate the matrix

$$
A(\pi)=\left(\begin{array}{cccc}
s_{1} & s_{2} & \cdots & s_{n} \\
t_{1} & t_{2} & \cdots & t_{n}
\end{array}\right)
$$


where $j_{0}=0$ and $s_{k}=r\left(j_{0}+\cdots+j_{r}+1 \leqq k \leqq j_{0}+\cdots+j_{r}+j_{r+1}\right)$ and $t_{k}$ is the weight assigned to the element $i_{k}$ when the permutation $\pi$ is read.

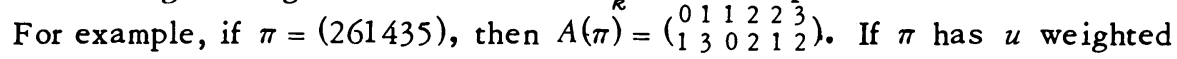
rises and $v$ weighted readings, then it is immediate that $u=\Sigma s_{i}$ and $v=$ $\Sigma t_{i}$. Thus, if $\alpha=\left(a_{1}, \ldots, a_{n}\right)$ and $\beta=\left(b_{1}, b_{2}, \ldots, b_{n}\right)$ are partitions of $k-u$ and $m-v$; i.e., if $\Sigma a_{i}=k-u, \Sigma b_{i}=m-v, 0 \leqq a_{i} \leqq a_{i+1}$, and $0 \leqq$ $b_{i} \leqq b_{i+1}$, then the matrix

$$
A(\pi ; \alpha, \beta)=A(\pi)+\left(\begin{array}{cccc}
a_{1} & a_{2} & \cdots & a_{n} \\
b_{i_{1}} & b_{i_{2}} & \cdots & b_{i_{n}}
\end{array}\right)=\left(\begin{array}{ccc}
a_{1}+s_{1} & \cdots & a_{n}+s_{n} \\
b_{i_{1}}+t_{1} & \cdots & b_{i_{n}}+t_{n}
\end{array}\right)
$$

is a partition of the bipartite $(k, m)$. We claim that each partition counted by $N_{n}(k, m)$ is obtained in this way.

Since $a_{i} \leqq a_{i+1}$ and $s_{i} \leqq s_{i+1}$, it is immediate that the first row of $A(\pi ; \alpha, \beta)$ is nondecreasing. Also these inequalities show that if $a_{j}+s_{j}=$ $a_{r}+s_{r}$ with $1 \leqq j<r \leqq n$ then $a_{j}=a_{r}$ and $s_{j}=s_{r}$. This last entails that $i_{j}>i_{r}$ and thus that the element $i_{r}$ is read on an earlier scan than the element $i_{j}$; i.e., $t_{j}>t_{r}$. On the other hand, since $i_{j}>i_{r}$, we have that $b_{i_{j}} \geqq b_{i_{r}}$ and so $b_{i_{j}}+t_{j}>b_{i_{r}}+t_{r}$, as desired.

In the next place, if $\pi=\left(i_{1}, \ldots, i_{n}\right), \pi^{\prime}=\left(j_{1}, \ldots, j_{n}\right)$ are permutations and $\alpha, \alpha^{\prime}$ and $\beta=\left(b_{1}, \cdots, b_{n}\right), \beta^{\prime}=\left(c_{1}, \ldots, c_{n}\right)$ are partitions such that $A(\pi ; \alpha, \beta)=A\left(\pi^{\prime} ; \alpha^{\prime}, \beta^{\prime}\right)$, then we must show that $\pi=\pi^{\prime}, \alpha=\alpha^{\prime}$, and $\beta=\beta^{\prime}$. We first note that the inequality $b_{i_{r}}+t_{r} \leqq b_{i_{r+1}}=\dot{t}_{r+1}$ is equivalent to $s_{r+1}=$ $1+s_{r}$ or, what is the same thing, $i_{r}<i_{r+1}$. Using this remark, we see that $\pi$ and $\pi^{\prime}$ have the same $s$ vector and that this requires that $\alpha=\alpha^{\prime}$. We now conclude the proof by induction on $n$. First choose $r$ such that $i_{r}=1$. Then $t_{r}=0$ and $b_{i_{r}}+t_{r}=b_{1}=c_{j_{r}}+t_{r}^{\prime} \geqq c_{1}$, where of course $t_{r}^{\prime}$ denotes the $r$ th entry in the reading vector for $\pi^{\prime}$. In the same way, we see that $c_{1} \geqq b_{1}$ and so $b_{1}=c_{1}$. With this same choice of $r$ we also note that $c_{1}=b_{1}=c_{j_{r}}+\dot{t}_{r}^{\prime}$ $\geqq c_{1}+t_{r}^{\prime}$ and so $t_{r}^{\prime}=0$. Thus the element $j_{r}$ is read on the first scan of $\pi^{\prime}$ and if $j_{r}>1$, there would be some integer $s<r$ for which $j_{s}=1$. However, since $i_{s}>1$, we have $c_{1}=b_{i_{s}}+t_{s}>b_{i_{s}} \geqq b_{1}=c_{1}$, a contradiction. Thus $j_{r}=1$ and by the inductive hypothesis we conclude that $\beta=\beta^{\prime}$ and $\pi=\pi^{\prime}$.

Finally, if

$$
\Gamma=\left(\begin{array}{lll}
c_{1} & \cdots & c_{n} \\
d_{1} & \cdots & d_{n}
\end{array}\right)
$$


is a partition of the bipartite $(k, m)$ counted by $N_{n}(k, m)$, we must construct a permutation $\pi$ and partitions $\alpha$ and $\beta$ such that $A(\pi ; \alpha, \beta)=\Gamma$. First order the second row of $\Gamma$ as $d_{j_{1}} d_{j_{2}} \cdots d_{j_{n}}$ so that $d_{r}$ precedes $d_{s}$ if either $d_{r}<d_{s}$ or $d_{r}=d_{s}$ and $r<s$. Define the permutation

$$
\pi=\left(\begin{array}{cccc}
1 & 2 & \cdots & n \\
i_{1} & i_{2} & \cdots & i_{n}
\end{array}\right) \text { by } \pi^{-1}=\left(\begin{array}{cccc}
1 & 2 & \cdots & n \\
j_{1} & j_{2} & \cdots & j_{n}
\end{array}\right)
$$

and let

$$
A(\pi)=\left(\begin{array}{ccc}
s_{1} & \cdots & s_{n} \\
t_{1} & \cdots & t_{n}
\end{array}\right)
$$

Then define the partitions $a=\left(a_{1}, \ldots, a_{n}\right)$ and $\beta=\left(b_{1}, \ldots, b_{n}\right)$ according to $a_{r}=c_{r}-s_{r}$ and $b_{r}=d_{j_{r}}-t_{j_{r}}$. It is immediate that $\Gamma=A(\pi ; \alpha, \beta)$ and it is only necessary to verify that $\alpha$ and $\beta$ defined in this way are partitions; i.e., that $0 \leqq a_{r} \leqq a_{r+1}$ and $0 \leqq b_{r} \leqq b_{r+1}$. These last inequalities follow rather easily. For example if $r$ is the smallest index such that $a_{r+1}<0$, then it follows that $c_{r}-s_{r}>c_{r+1}-s_{r+1}$ and from $c_{r} \leqq c_{r+1}$ and $s_{r+1} \leqq 1+$ $s_{r}$, we obtain $c_{r}=c_{r+1}$ and $s_{r+1}=1+s_{r}$. However, since $c_{r}=c_{r+1}$, we have $d_{r}>d_{r+1}$ so that $i_{r}>i_{r+1}$ and this requires that $s_{r}=s_{r+1}$, a contradiction. The proof that $a_{r} \leqq a_{r+1}$ is identical and the proof that $\beta$ is a partition is similar.

3. Computations. The combinatorial meaning assigned to the coefficients $d_{n}(u, v)$ by $(1.6)$ makes it possible to compute their values for small values of $n$. The following tables indicate these values for $4 \leqq n \leqq 6$.

\begin{tabular}{l|lllllll}
$u \backslash v$ & 0 & 1 & 2 & 3 & 4 & 5 & 6 \\
\hline 0 & 1 & & & & & & \\
1 & & 1 & 1 & 1 & & & \\
2 & & 1 & 2 & 1 & 1 & & \\
3 & & 1 & 1 & 2 & 1 & 1 & \\
4 & & & 1 & 1 & 2 & 1 & \\
5 & & & & 1 & 1 & 1 & \\
6 & & & & & & & 1
\end{tabular}

$$
n=4
$$




\begin{tabular}{c|ccccccccccc}
$u \backslash v$ & 0 & 1 & 2 & 3 & 4 & 5 & 6 & 7 & 8 & 9 & 10 \\
\hline 0 & 1 & & & & & & & & & & \\
1 & & 1 & 1 & 1 & 1 & & & & & & \\
2 & & 1 & 2 & 2 & 2 & 1 & 1 & & & & \\
3 & & 1 & 2 & 3 & 3 & 3 & 2 & 1 & & & \\
4 & & 1 & 2 & 3 & 4 & 4 & 3 & 2 & 1 & & \\
5 & & & 1 & 3 & 4 & 6 & 4 & 3 & 1 & & \\
6 & & & 1 & 2 & 3 & 4 & 4 & 3 & 2 & 1 & \\
7 & & & & 1 & 2 & 3 & 3 & 3 & 2 & 1 & \\
8 & & & & & 1 & 1 & 2 & 2 & 2 & 1 & \\
9 & & & & & & & 1 & 1 & 1 & 1 & \\
10 & & & & & & & & & & & 1
\end{tabular}

$$
n=5
$$

\begin{tabular}{c|cccccccccccccccc}
$u \backslash v$ & 0 & 1 & 2 & 3 & 4 & 5 & 6 & 7 & 8 & 9 & 10 & 11 & 12 & 13 & 14 & 15 \\
\hline 0 & 1 & & & & & & & & & & & & & & & \\
1 & & 1 & 1 & 1 & 1 & 1 & & & & & & & & & \\
2 & & 1 & 2 & 2 & 3 & 2 & 2 & 1 & 1 & & & & & & \\
3 & & 1 & .2 & 4 & 4 & 5 & 5 & 4 & 2 & 2 & & & & & \\
4 & & 1 & 3 & 4 & 7 & 7 & 8 & 7 & 6 & 3 & 2 & 1 & & & & \\
5 & & 1 & 2 & 5 & 7 & 11 & 11 & 12 & 9 & 7 & 4 & 2 & & & \\
6 & & & 2 & 5 & 8 & 11 & 15 & 14 & 13 & 10 & 7 & 3 & 2 & & & \\
7 & & & 1 & 4 & 7 & 12 & 14 & 17 & 15 & 13 & 9 & 6 & 2 & 1 & & \\
8 & & & 1 & 2 & 6 & 9 & 13 & 15 & 17 & 14 & 12 & 7 & 4 & 1 & & \\
9 & & & & 2 & 3 & 7 & 10 & 13 & 14 & 15 & 11 & 8 & 5 & 2 & & \\
10 & & & & & 2 & 4 & 7 & 9 & 12 & 11 & 11 & 7 & 5 & 2 & 1 & \\
11 & & & & & 1 & 2 & 3 & 6 & 7 & 8 & 7 & 7 & 4 & 3 & 1 & \\
12 & & & & & & & 2 & 2 & 4 & 5 & 5 & 4 & 4 & 2 & 1 & \\
13 & & & & & & & & 1 & 1 & 2 & 2 & 3 & 2 & 2 & 1 & \\
14 & & & & & & & & & & & 1 & 1 & 1 & 1 & 1 & \\
15 & & & & & & & & & & & & & & & & 1
\end{tabular}

$$
n=6
$$




\section{REFERENCES}

1. R. Bellman, Bull. Amer. Math. Soc. 61 (1955), 92. Problems 1-5.

2. L. Carlitz, The expansion of certain products, Proc. Amer. Math. Soc. 7 (1956), 558-564. MR 19, 29.

3. L. Carlitz, D. P. Roselle and R. A. Scoville, Permutations and sequences with repetitions by number of increases, J. Combinatorial Theory 1 (1966), 350-374. MR 34 \#77.

4. B. Gordon, Two theorems on multipartite partitions, J. London Math. Soc. 38 (1963), 459-464. MR 28 \#1187.

5. Richard P. Stanley, Ordered structures and partitions, Mem. Amer. Math. Soc. No. 119 (1972).

6. - The conjugate trace and trace of a plane partition, J. Combinatorial Theory 14 (1973), 53-65.

7. E. M. Wright, Partitions of multipartite numbers, Proc. Amer. Math. Soc. 7 (1956), 880-890. MR 18, 793.

8. - Partitions of multipartite numbers into a fixed number of parts, Proc. London Math. Soc. (3) 11 (1961), 499-510. MR 24 \#A2573.

DEPARTMENT OF MATHEMATICS, LOUISIANA STATE UNIVERSITY, BATON ROUGE, LOUISIANA 70803 